\title{
A Vehicle Haptic Steering by Wire System Based on High Gain GPI Observers
}

\author{
A. Rodriguez-Angeles, ${ }^{1,2}$ H. Sira-Ramirez, ${ }^{1}$ and J. A. Garcia-Antonio ${ }^{1}$ \\ ${ }^{1}$ Center for Research and Advanced Studies (CINVESTAV-IPN), Electrical Engineering Department, Mechatronics Group, \\ Avenida IPN, No. 2508, Col. San Pedro Zacatenco, 07630 Mexico City, DF, Mexico \\ ${ }^{2}$ Dynamics and Control Group, Department of Mechanical Engineering, Eindhoven University of Technology, P.O. Box 513, \\ 5600 MB Eindhoven, The Netherlands
}

Correspondence should be addressed to A. Rodriguez-Angeles; aangeles@cinvestav.mx

Received 12 November 2013; Accepted 31 March 2014; Published 30 June 2014

Academic Editor: Ilse Cervantes

Copyright (C) 2014 A. Rodriguez-Angeles et al. This is an open access article distributed under the Creative Commons Attribution License, which permits unrestricted use, distribution, and reproduction in any medium, provided the original work is properly cited.

\begin{abstract}
A vehicle steering by wire (SBW) haptic system based on high gain generalized proportional integral (GPI) observers is introduced. The observers are considered for the estimation of dynamic perturbations that are present at the tire and steering wheel. To ensure efficient tracking between the commanded steering wheel angle and the tire orientation angle, the estimated perturbations are on line canceled. As to provide a haptic interface with the driver, the estimated dynamic effects at the steering rack are fed back to the steering wheel, yielding a master-slave haptic system with bilateral communication. For implementation purposes few sensors and minimum knowledge of the dynamic model are required, which is a major advantage compared to other approaches. Only position tracking errors are fed back, while all other signals are estimated by the high gain GPI observers. The scheme is robust to uncertainty on the input gain and cancels dynamic perturbation effects such as friction and aligning forces on the tire. Experimental results are presented on a prototype platform.
\end{abstract}

\section{Introduction}

Automobile dynamics, safety, and comfort are taking a major role in vehicle design [1]. The steering system, or rather the interface between the driver and the vehicle, is becoming an important part of automobile design tasks [2-4]. The basic design of the automobile steering system has changed little since the steering wheel invention. The driver commanded direction is transmitted by a column or steering shaft, including universal joints and gearboxes, to the front tires. Assisted steering systems were introduced in 1950, with the hydraulic power steering system. Since then, the assistance unit has become standard in modern vehicles. The steering system complements the effort required by the driver to steer the vehicle, by using hydraulic pressure. Meanwhile, electrical assisted steering systems have been introduced recently, which eliminate the requirement of a hydraulic pump or diminish the required effort provided by the hydraulic power, yielding electrohydraulic or electrical assistance to the steering rack $[5,6]$.
The steering systems can be classified into three main groups: mechanical steering systems [7], hydraulic power and electrohydraulic power systems, and electric power assisted systems (EPAS) [8-10]. Among the EPAS, there is a new technology called steering by wire (SBW), whose main characteristic is to completely do away with as many mechanical components (steering shaft, column, gear reduction mechanism, etc.) as possible [11]; see Figure 1.

In general SBW systems exhibit several advantages, such as the following.

(1) The absence of steering column simplifies the car interior design.

(2) The absence of steering shaft, column and gear reduction mechanism, allows much better space utilization in the engine compartment.

(3) The steering mechanism can be designed and installed as a modular unit. 


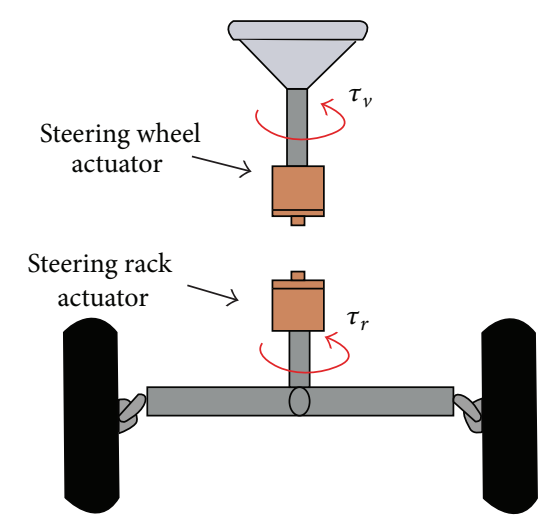

(a)

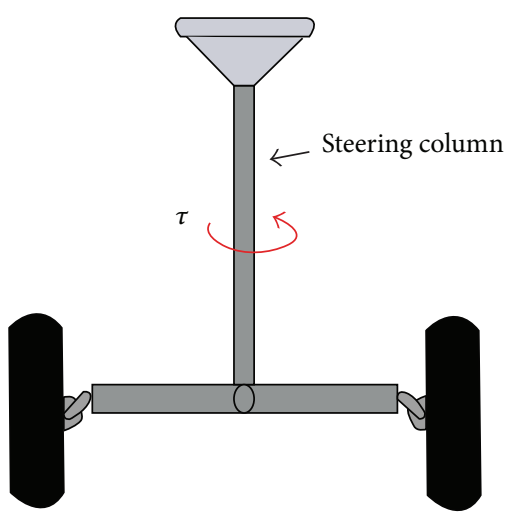

(b)

FIGURE 1: (a) SBW; (b) conventional steering system.

(4) Without mechanical connection between the steering wheel and the road wheel, it is less likely that the impact of a frontal crash will force the steering wheel to invade into the driver's survival space.

(5) SBW system characteristics can easily be adjusted to optimize the steering response and driving feeling [12, 13].

Therefore, SBW systems allow better structures for crash energy absorption [14] and present benefits related to passengers' comfort and driver feeling.

While substituting conventional steering systems by SBW, there is a major issue related to what the driver feels through the steering wheel. The forces, torques, and driving conditions transmitted by the steering wheel to the driver are important for a proper and safe vehicle driving. Therefore, the SBW must provide the driver the opportunity to "feel" what the road driving conditions are, just as the steering column does. As a consequence, the SBW mechanism must also behave as a haptic device [13,14].

Several dynamic models related to haptic SBW systems are presented in the literature; see [13-16]. In these models, uncertain and perturbation terms appear which affect the dynamics and the behavior of the SBW. Phenomena such as friction, damping, inertia, and aligning forces, among others, are considered in the dynamic model. The influence of those uncertain and perturbation terms is rather important to guarantee a good performance of the SBW and to provide a reliable feedback of the road and driving conditions to the driver. Dynamic phenomena effects on the SBW are difficult to be known with certainty due to the changing conditions on the road and the driving, for example, speed, roadway texture, tire wear, tire air pressure, and rain. For the purpose of estimating the effects of such unknown perturbations, the use of observers has been proposed [11, 17]. The implementation of such observers often requires the use of specialized sensors, such as GPS and INS, measurement of lateral acceleration [12], and current and torque motors measurements [15]. Furthermore, most of the proposed observers highly rely on complicated dynamic models of the phenomena that are present in the steering system. Besides uncertainty on the dynamics phenomena, there exists also the problem of model changes in accordance with the driving conditions and the wide variety of vehicle models $[1,16]$.

All that was previously described makes it difficult to determine a precise mathematical model and a control strategy for the SBW system. As a solution, model-free observers might be considered for estimating unmodeled dynamics and perturbations effects on SBW systems.

In this work a vehicle SBW system is proposed based on high gain generalized proportional integral (GPI) observers. The use of extended and high gain observers is rather common in active disturbance rejection control (ADRC), [18, 19], and some applications of such approach can be revised in [20-22].

In the proposed approach high gain GPI observer helps in determining the effects of uncertain dynamics phenomena and additive perturbations on the steering rack and tire, such as continuous and discontinuous friction, aligning forces, inertia effects, and damping. The purpose of the high gain GPI observer is twofold: firstly to help a PD controller to actively reject dynamic perturbations and secondly to provide the forces and torques that are fed back to the driver through the steering wheel, therefore closing a haptic loop. The proposed approach is only based on angle position feedback; therefore, it can be implemented with common low cost encoder sensors as in the experimental platform here considered.

The proposed SBW system behaves as a self-contained sensor for tire forces, since such forces directly influence vehicle dynamics. Although all uncertainties and dynamic perturbations are lumped into an estimated linear term, the approach shows good performance and a proper estimation of the combined dynamic effects that are present at the SBW system. Experimental results on a real platform show good agreement with the theoretical results, that allows concluding convergence of the tracking and estimation errors to a small vicinity around zero.

\section{SBW Dynamic Model}

When considering a vehicle dynamics there are several involved phenomena, such as lateral and longitudinal forces, 
tire-road friction $[16,23]$. A common approach on vehicle modeling dynamics is the so-called bicycle model $[12,16,24]$, which considers the chassis. However for the purpose of this paper and taking into account that the experimental platform only considers one tire (half of the steering rack), only the steering system dynamics is to be taken into account.

As result of the SBW design of the experimental platform, the steering wheel system depicted in Figure 3 and the steering rack system in Figure 5 are very similar. Thus, based on their similarities, the dynamic model of the steering rack subsystem is obtained alike that of the steering wheel. But, at the steering rack system the tire-road dynamic phenomena are included, such as tire-road interaction and aligning forces.

For modeling, control, and implementation purposes the SBW system might be viewed as a master-slave system with bilateral communication. In such approach, the steering wheel acts as a master subsystem, capturing the driver commanded steering angle, and the steering rack is seen as a slave subsystem that has to track the operator commanded steer angle. The bilateral communication implies that the steering wheel sends the commanded angle to the steering rack, while from the steering rack, dynamic effects are estimated and sent to the steering wheel subsystem. Furthermore each subsystem is provided with a $\mathrm{PD}$ controller with active disturbance rejection and high gain GPI observers. The overall system thus conforms a bilateral control scheme as in [24].

2.1. Steering Wheel Subsystem. The dynamics of the steering wheel (Figure 2) or master system are affected by physical phenomena that are presented in its three fundamental components: the wheel, the reduction gearbox, and the DC motor (Figure 3). In particular the DC motor is in charge of reflecting to the driver the forces that are estimated by the high gain GPI observer from the dynamical phenomena presented on the steering rack and tire (slave subsystem). Uncertain dynamic effects and perturbation on the wheel subsystem are estimated by another high gain GPI observer and canceled through a PD controller with disturbance rejection. Dynamic effects on the steering wheel include discontinuous Coulomb friction; other works such as $[12,24]$ considered that Coulomb friction phenomena affect only the steering rack and tire subsystem. However the use of a reduction gearbox induces discontinuous friction phenomena, even dead zones may occur depending on the reduction gear.

Figure 3 shows an equivalent mechanical-electrical diagram of the steering wheel subsystem. It is shown how the dynamics of its three components are interconnected. For the electrical part the relevant parameters are $V_{v}$ the input voltage, $L_{v}$ armature inductance, $R_{v}$ armature resistance, $i_{v}$ armature current, $i_{f}$ current through the winding field, $e_{v}$ electromotive force, and $k_{2}$ electromotive force constant gain. Meanwhile, the mechanical relevant parameters are $\phi_{v}$ output angle of the motor, $J_{1}$ inertia moment at the reduction input, $B_{1}$ damping coefficient at the reduction input, $F_{1}$ Coulomb friction coefficient at the reduction input, $k_{1}$ motor torque constant gain, $\tau_{v^{\prime}}$ motor torque, $F_{T v}$ Coulomb friction coefficient at gearbox, $B_{T v}$ damping coefficient at gearbox, $J_{2}$ inertia moment at the reduction output, $B_{2}$ damping coefficient at

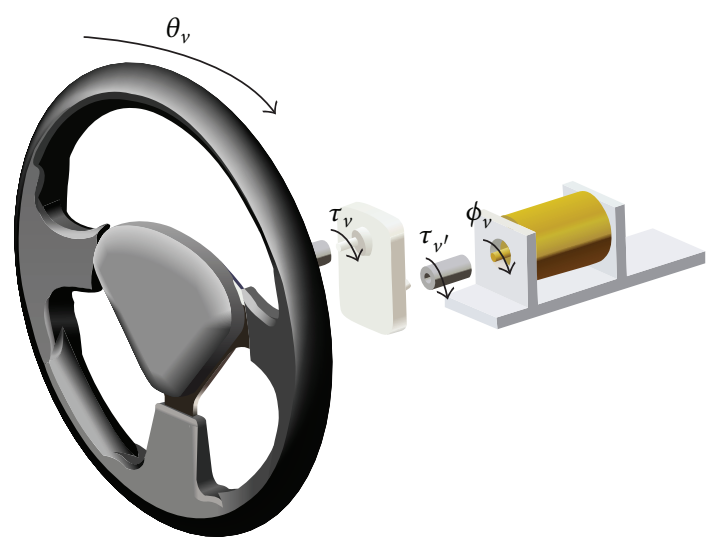

FIGURE 2: Steering wheel subsystem.

the reduction output, $F_{2}$ Coulomb friction coefficient at the reduction output, $\tau_{v}$ torque at the reduction output, $C_{v}$ wheel Coulomb friction coefficient, $B_{v}$ wheel damping coefficient, $J_{v}$ wheel inertia moment, $N_{v}=N_{v 1} / N_{v 2}$ gearbox reduction ratio, $N_{v 1}$ the number of teeth on the input gear, $N_{v 2}$ the number of teeth on the output gear, and $\theta_{v}$ wheel angular position.

For the electrical part of the steering wheel system, the dynamic relation between the torque at the reduction output $\tau_{v}$ and the input voltage $V_{v}$ is given by

$$
\tau_{v}=\frac{N_{v} k_{1}}{R_{v}} V_{v}(t)-\frac{N_{v}^{2} k_{1} k_{2}}{R_{v}} \dot{\theta}_{v} .
$$

Meanwhile, taking into account the reduction gearbox, the dynamic relation between $\tau_{v}$ and the wheel angular position $\theta_{v}$ is represented by

$$
\tau_{v}=J_{T v} \ddot{\theta}_{v}+B_{T v} \dot{\theta}_{v}+F_{T v} \operatorname{sign}\left(\dot{\theta}_{v}\right),
$$

where the equivalent coefficients of the reduction gearbox are given by

$$
\begin{aligned}
J_{T v} & =\left(\frac{N_{v 2}}{N_{v 1}}\right)^{2} J_{1}+J_{2}, \\
B_{T v} & =\left(\frac{N_{v 2}}{N_{v 1}}\right)^{2} B_{1}+B_{2}, \\
F_{T v} & =\left(\frac{N_{v 2}}{N_{v 1}}\right)^{2} F_{1}+F_{2} .
\end{aligned}
$$

Finally, taking into account (1) and (2) and the dynamic effects of the steering wheel, it is obtained that the dynamic model of the steering wheel subsystem is given by (4), with $J_{v e}=J_{v}+J_{T v}$ being the equivalent inertia of the steering wheel system:

$$
\begin{gathered}
J_{v e} \ddot{\theta}_{v}+\left(B_{T v}+B_{v}\right) \dot{\theta}_{v}+\left(F_{T v}+C_{v}\right) \operatorname{sign}\left(\dot{\theta}_{v}\right) \\
=\frac{N_{v} k_{1}}{R_{v}} V_{v}(t)-\frac{N_{v}^{2} k_{1} k_{2}}{R_{v}} \dot{\theta}_{v} .
\end{gathered}
$$




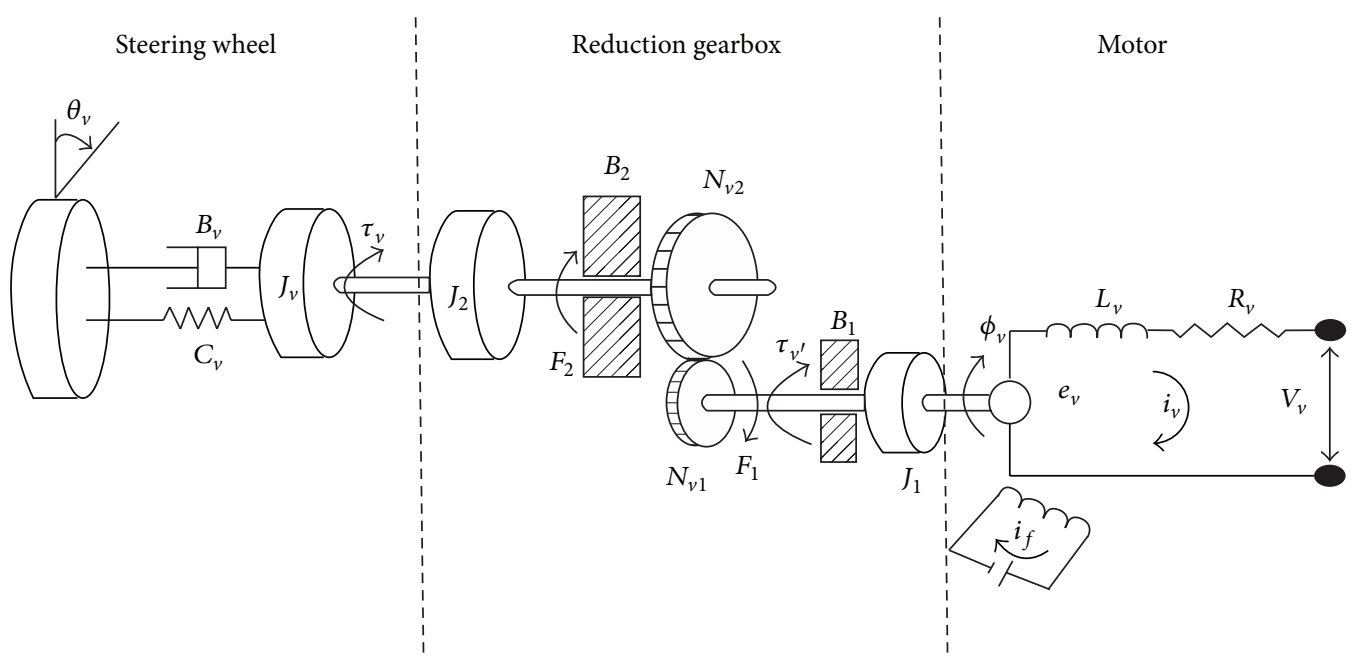

Figure 3: Equivalent mechanical-electrical diagram of the steering wheel subsystem.

2.2. Steering Rack Subsystem and Tire Dynamics. Figure 4 shows the steering rack and tire subsystem (slave subsystem), while a schematic diagram is presented in Figure 5, where the main components (tire, reduction gearbox, and DC motor) are represented by its electrical and mechanical counterparts. Notice the similarities between the steering wheel subsystem depicted in Figure 3 and the steering rack subsystem in Figure 5. Thus, based on their similarities, the dynamic model of the steering rack subsystem is obtained alike that of the steering wheel. However, because the tire-road interaction, there are dynamic effects such as friction and aligning forces that must to be considered [16, 17]. Also longitudinal forces affect the tire dynamics, but at this point, such forces are disregarded and would be further included when vehicle chassis is to be considered.

From Figure 5 and by taking into account the electrical and mechanical parameters, the dynamic model of the steering rack and tire subsystem is obtained as given by (5). The aligning forces at the tire, also referred to as self-aligning forces in the literature, are represented by $\tau_{\mathrm{SAT}}$. Meanwhile, the relevant electrical parameters in model (5) are $V_{r}$ the input voltage, $L_{r}$ armature inductance, $R_{r}$ armature resistance, $i_{r}$ armature current, $i_{f}$ current through the winding field, $e_{r}$ electromotive force, and $k_{2}$ electromotive force constant gain. For the mechanical part, the parameters are $\phi_{r}$ output angle of the motor, $J_{a}$ inertia moment at the reduction input, $B_{a}$ damping coefficient at the reduction input, $F_{a}$ Coulomb friction coefficient at the reduction input, $k_{1}$ motor torque constant gain, $F_{T r}$ Coulomb friction coefficient at gearbox, $B_{T r}$ damping coefficient at gearbox, $\tau_{r^{\prime}}$ motor torque, $J_{b}$ inertia moment at the reduction output, $B_{b}$ damping coefficient at the reduction output, $F_{b}$ Coulomb friction coefficient at the reduction output, $\tau_{r}$ rack torque, $C_{r}$ rack Coulomb friction coefficient, $B_{r}$ rack damping coefficient, $J_{r}$ rack inertia moment, $N_{r}=N_{r 1} / N_{r 2}$ gearbox reduction ratio, $N_{r 1}$ the number of teeth on the input gear, $N_{r 2}$ the number of teeth on the output gear, $\theta_{r}$ rack angular position (tire angular position), and $J_{r e}=J_{r}+J_{T r}$ the equivalent inertia of the steering rack subsystem:

$$
\begin{gathered}
J_{r e} \ddot{\theta}_{r}+\left(B_{T r}+B_{r}\right) \dot{\theta}_{r}+\left(F_{T r}+C_{r}\right) \operatorname{sign}\left(\dot{\theta}_{r}\right)+\tau_{\mathrm{SAT}} \\
=\frac{N_{r} k_{1}}{R_{r}} V_{r}(t)-\frac{N_{r}^{2} k_{1} k_{2}}{R_{r}} \dot{\theta}_{r} .
\end{gathered}
$$

The equivalent coefficients of the rack reduction gearbox are given by

$$
\begin{gathered}
J_{T r}=\left(\frac{N_{r 2}}{N_{r 1}}\right)^{2} J_{a}+J_{b}, \\
B_{T r}=\left(\frac{N_{r 2}}{N_{r 1}}\right)^{2} B_{a}+B_{b}, \\
F_{T r}=\left(\frac{N_{r 2}}{N_{r 1}}\right)^{2} F_{a}+F_{b} .
\end{gathered}
$$

2.2.1. Aligning Force at Tire. The aligning torque $\tau_{\mathrm{SAT}}$ occurs because of the existence of caster and kingpin angles in the steering mechanical structure; this torque induces steering pull. Both linear and nonlinear models for lateral aligning forces have been proposed, mainly based on cornering stiffness, the slip angle, and the so-called bicycle model of a front steering vehicle $[16,24]$. While a vehicle is turning, tire forces acting on the steering system tend to resist steering motion away from the straight-ahead position. These forces are due to self-aligning torque, that is a function of the steering geometry, particularly caster angle, tire deformation, and vehicle linear velocity. Since the considered experimental platform is only composed by half of the steering rack (see Figure 8), then only one tire is taken into account.

The aligning torque at the front left wheel is modeled as presented in [23]. Figure 6 depicts the forces and variables related to aligning tire torque. The subindex $f l$ indicates that 


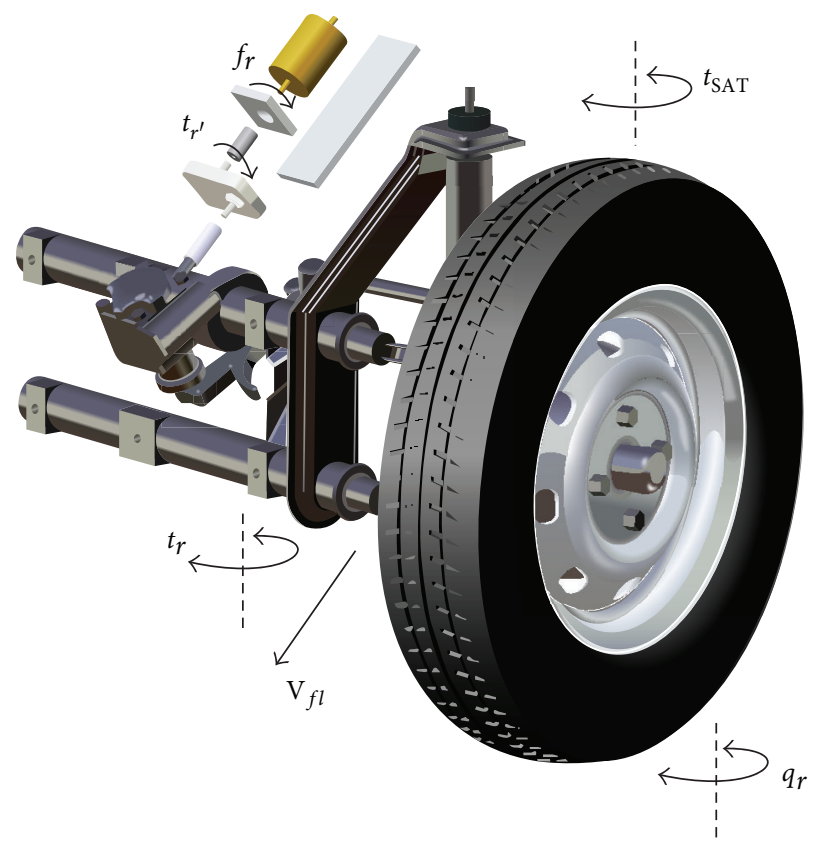

FIGURE 4: Steering rack and tire subsystem.

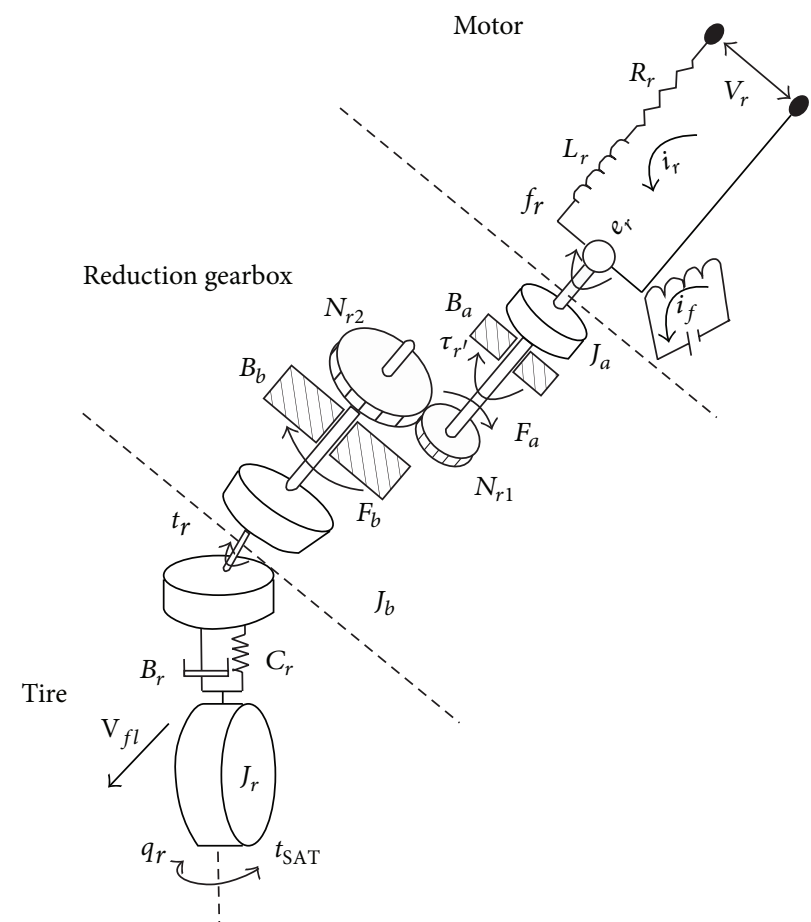

FIGURE 5: Equivalent mechanical-electrical diagram of the steering rack and tire subsystem. 


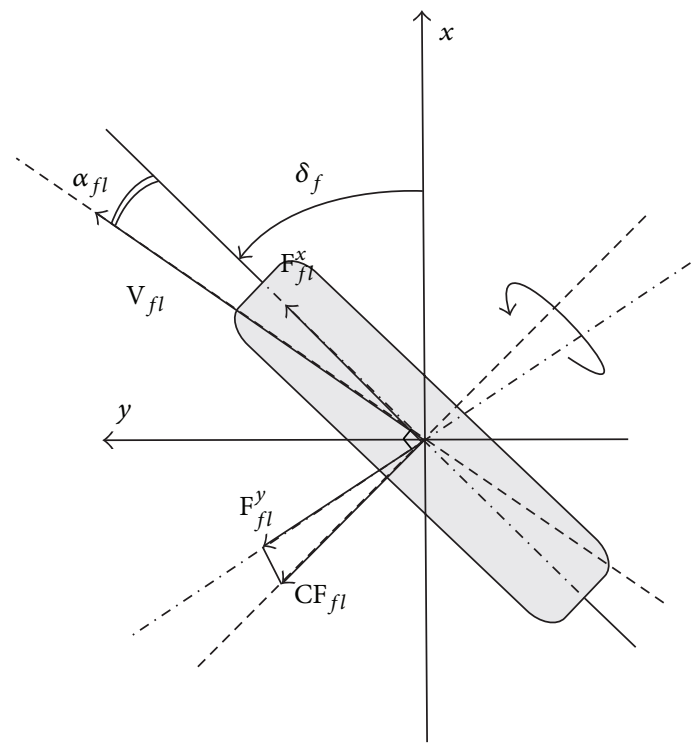

(a)

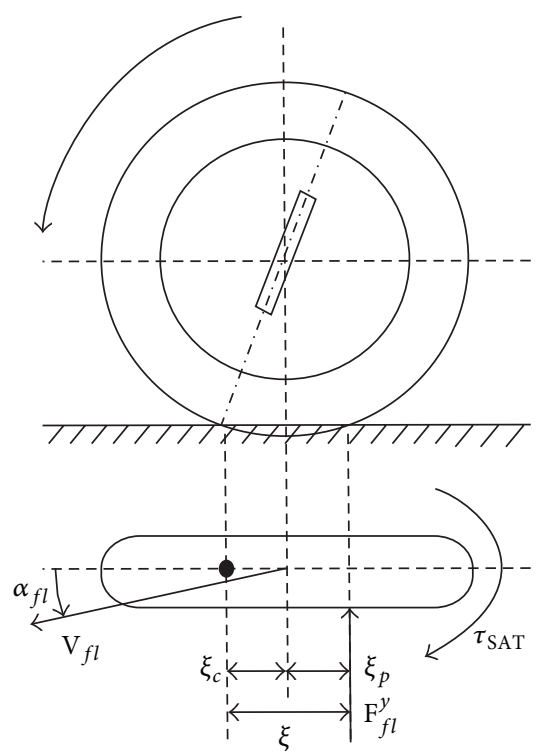

(b)

FIgURE 6: Tire force at front left wheel: (a) tire forces; (b) self-aligning torque [23].

front left tire is considered, while the superindices $x$ and $y$ reflect the axes on which forces act. $F^{x}$ and $F^{y}$ are driving and lateral forces, respectively. $F^{y}$ is generated by sidesliping of the tire. $C F$ stands for cornering force. $F_{f l}^{y}$ is the front left tire lateral force, $\alpha_{f l}$ is the front tire slip angle, $\xi_{p}$ is the pneumatic trail, that is, the distance from the center of tire to the application of lateral force, $\xi_{c}$ is the caster or mechanical trail, that is, the distance from the tire center to the point on the ground about which the tire pivots as a result of the wheel caster angle, and $V_{f l}$ is the velocity of the tire at its center. Then the self-aligning torque is given by

$$
\tau_{\mathrm{SAT}}=\left(\xi_{c}+\xi_{p}\right) F_{f l}^{y}\left(\alpha_{f l}\right) .
$$

According to [23], in the linear region of tire characteristics, the front left tire cornering force $C F_{f l}$ depends on cornering stiffness $C_{f}$ and the slipping angle $\alpha_{f l}$; that is,

$$
C F_{f l}=-C_{f} \alpha_{f l} .
$$

Then from Figure 6, the relation between $F_{f l}^{y}$ and $C F_{f l}$ is given by

$$
F_{f l}^{y}=\frac{C F_{f l}}{\cos \alpha_{f l}} .
$$

Note that the tire slip angle $\alpha_{f l}$ is not easy to be measured; thus it must be estimated. In [12], it is mentioned that on production cars' sideslip angle is typically derived from integration of inertial sensors (lateral acceleration), but this estimation error is prone to uncertainty and errors due to sensor drift. Furthermore, $\xi_{c}$ and $\xi_{p}$ are only approximately known; however, in order to obtain a linear model, $\xi_{c}$ and $\xi_{p}$ are assumed to be constant and known. Same consideration applies to the front cornering stiffness $C_{f}$.
Remark 1. Notice that the dynamic models here represented by (4) and (5) are not used for the design of the PD controller and the high gain GPI observers. The only parameter that is required for the high gain GPI observer is the input gain. Nevertheless, the dynamic models are introduced to show the dynamic phenomena that affect the SBW system.

\section{PD plus High Gain GPI Observer}

The design of the controller and observer for both the steering wheel and the steering rack is very similar, but for the sake of clarity, each controller and observer would be presented separately.

3.1. Steering Wheel Controller and Observer. Based on the dynamic model of the steering wheel or master subsystem (4), the nonlinear terms, including perturbations and unmodeled dynamics, are substituted by a time-variant disturbance term $\varphi(t)$. Lumping all uncertainties and unknown dynamics on the time-varying term $\varphi(t)$ might be considered as a rough approximation; nevertheless under certain conditions on boundedness and smoothness such approximation results are valid (see the appendix and $[20,21])$. In state space dynamics representation, $x_{1}=\theta_{v}$ and $x_{2}=\dot{\theta}_{v}$; the model (4) is represented as a linear perturbed system depicted in

$$
\begin{gathered}
\dot{x}_{1}=x_{2}, \\
\dot{x}_{2}=\frac{N_{v} k_{1}}{J_{v e} R_{v}} V_{v}(t)+\varphi(t),
\end{gathered}
$$

where $\varphi(t)$ is by assumption a uniformly, absolutely, bounded, time-varying function, that represents all nonlinear and 
uncertain terms, that is, all disturbances affecting the linear simplified model (see (11)):

$$
\begin{aligned}
\varphi(t)=-\frac{1}{J_{v e}}\{ & \left(B_{T v}+B_{v}\right) \dot{\theta}_{v} \\
& \left.+\left(F_{T v}+C_{v}\right) \operatorname{sign}\left(\dot{\theta}_{v}\right)+\frac{N_{v}^{2} k_{1} k_{2}}{R_{v}} \dot{\theta}_{v}\right\} .
\end{aligned}
$$

Note that the equality established by (11) is enforced by the proposed model (4); if other dynamic effects or perturbations are to be considered, these phenomena would be included in the term $\varphi(t)$ as well, such that the approximated linear model (10) remains valid in local basis. The relation (11) is presented for the sake of completeness, since the term $\varphi(t)$ is estimated by a high gain GPI observer.

Based on the state space representation (10), a high gain GPI observer is proposed for the linear perturbed system, given by (12). The number of integrators required at the high gain GPI observer depends on the order and complexity of the nonlinearities represented by the term $\varphi(t)$. This is equivalent to truncating a Taylor or Fourier series expansion, but in this case perfect approximation would imply an infinite integrators chain. Proper determination of the order of the extended observer would imply knowledge of the nonlinearities of the term $\varphi(t)$ as shown in [25]. The order of the observer and the relation between the high gain and the final estimation bound have been analyzed for some particular systems and under certain considerations [18, 19, 25]. Despite the difficulties in determining the order of the extended observer from a theoretical point of view, for some practical situations they can be adjusted by looking after an approximated model and measurements on the systems. In this particular case the number of integrators was adjusted by trial and error based on the value of the estimation error $\widetilde{e}_{v}=$ $x_{1}-\widehat{x}_{1}$ of a set of experiments. The term $\widehat{\varphi}_{1}$ corresponds to an estimate of the disturbance term $\varphi(t)$. Notice that the high gain GPI observer (12) only requires measurement of the steering wheel angle $x_{1}=\theta_{v}$ minimizing the amount of sensors on the system:

$$
\begin{aligned}
& \dot{\hat{x}}_{1}=\widehat{x}_{2}+\lambda_{7 v}\left(x_{1}-\widehat{x}_{1}\right), \\
& \dot{\hat{x}}_{2}=\frac{N_{v} k_{1}}{J_{v e} R_{v}} V_{v}(t)+\widehat{\varphi}_{1}+\lambda_{6 v}\left(x_{1}-\widehat{x}_{1}\right), \\
& \dot{\hat{\varphi}}_{1}=\widehat{\varphi}_{2}+\lambda_{5 v}\left(x_{1}-\widehat{x}_{1}\right), \\
& \dot{\hat{\varphi}}_{2}=\widehat{\varphi}_{3}+\lambda_{4 v}\left(x_{1}-\widehat{x}_{1}\right), \\
& \dot{\hat{\varphi}}_{3}=\widehat{\varphi}_{4}+\lambda_{3 v}\left(x_{1}-\widehat{x}_{1}\right), \\
& \dot{\hat{\varphi}}_{4}=\widehat{\varphi}_{5}+\lambda_{2 v}\left(x_{1}-\widehat{x}_{1}\right), \\
& \dot{\hat{\varphi}}_{5}=\widehat{\varphi}_{6}+\lambda_{1 v}\left(x_{1}-\widehat{x}_{1}\right), \\
& \dot{\hat{\varphi}}_{6}=\lambda_{0 v}\left(x_{1}-\widehat{x}_{1}\right) .
\end{aligned}
$$

The estimation error of the high gain GPI observer, $\tilde{e}_{v}$, satisfies the perturbed dynamics given by (13), which corresponds to a nonhomogeneous linear dynamic system, with input given by the sixth time derivative of $\varphi$. Similar convergence analyses are done in $[18,19,25]$, where there are also considerations on boundedness and smoothness of the perturbations and uncertainties, as those imposed here on the $\operatorname{term} \varphi$ :

$$
\tilde{e}_{v}^{(8)}+\lambda_{7 v} \widetilde{e}_{v}^{(7)}+\cdots+\lambda_{2 v} \ddot{\tilde{e}}_{v}+\lambda_{1 v} \dot{\tilde{e}}_{v}+\lambda_{0 v} \widetilde{e}_{v}=\varphi^{(6)} .
$$

Since $\varphi$ is by assumption a uniformly, absolutely, bounded, and time-varying function, then, to achieve convergence of the error dynamics to a small vicinity around the origin of the estimation error phase coordinates, the gains $\lambda_{i v}$, $i=0,1,2, \ldots, 7$ are chosen such that the roots of the associated characteristic polynomial are located far enough into the left half of the complex plane, that usually implies high gain values. From the results reported on ADRC, see [18, 19], it follows that closed loop stability properties can be established by considering slow and fast dynamics, usually of the control and the observer, respectively. This is, the observer dynamics should be faster than those of the controller, and for that a pole location problem can be considered based on (13). Among all possibilities to select the gains $\lambda_{i v}, i=$ $0,1,2, \ldots, 7$, a simple selection of the gains is done by assigning the associated characteristic polynomial to a suitable polynomial with known adequate roots. Since the order of the high gain observer error dynamics (13) is an even number, then a power of a second-order polynomial might be considered, as shown in (14), with $\xi_{\text {obs }, v}$ and $\omega_{n(\mathrm{obs}, v)}$ being coefficients related to damping and natural frequency, respectively:

$$
\begin{gathered}
s^{8}+\lambda_{7 v} s^{7}+\lambda_{6 v} s^{6}+\cdots+\lambda_{2 v} s^{2}+\lambda_{1 v} s+\lambda_{0 v} \\
=\left(s^{2}+2 \xi_{\text {obs }, v} \omega_{n(\mathrm{obs}, v)} s+\omega_{n(\mathrm{obs}, v)}^{2}\right)^{4} .
\end{gathered}
$$

Given that the observer (12) explicitly estimates the perturbation term $\varphi(t)$, then it is possible to propose a PD control with disturbance rejection (15). $\dot{\theta}_{v}^{*}(t)$ and $\ddot{\theta}_{v}^{*}(t)$ are the desired angular velocity and acceleration on the steering wheel, which might be obtained from the corresponding time derivatives of the angular position imposed by the driver $\theta_{v}^{*}(t)$, although this might raise problems due to noise:

$$
\begin{aligned}
V_{v}(t)=-\frac{J_{v e} R_{v}}{k_{1} N_{v}}[ & \widehat{\varphi}_{1}+\omega_{n(c, v)}^{2}\left(\theta_{v}-\theta_{v}^{*}\right) \\
& \left.+2 \xi_{c, v} \omega_{n(c, v)}\left(\widehat{x}_{2}-\dot{\theta}_{v}^{*}\right)-\ddot{\theta}_{v}^{*}+K_{h} \widehat{\sigma}_{1}\right] .
\end{aligned}
$$

Notice that in (15), $\widehat{x}_{2}$ corresponds to the estimate of the steering wheel angular velocity $\dot{\theta}_{v}(t)$, and $\widehat{\varphi}_{1}$ is the estimated disturbance term acting on the steering wheel subsystem $\varphi(t)$, so that both estimates are simultaneously obtained by the high gain GPI observer (12). Meanwhile, the term $\widehat{\sigma}_{1}$ corresponds to the estimate of the disturbance signal on the steering rack subsystem and it is also obtained by a high gain GPI observer designed for such particular system (19).

By defining the tracking error at the steering wheel subsystem as $e_{v}=\theta_{v}-\theta_{v}^{*}$, then the closed loop dynamics formed 
by (10) with the controller (15) and the high gain observer (12) are given by the nonhomogeneous linear equation (16). Once more following the results from $[18,19]$, selection of the control gains should imply that the observer error dynamics (13) must be faster than the controller dynamics:

$$
\begin{aligned}
\ddot{e}_{v} & +2 \xi_{c, v} \omega_{n(c, v)} \dot{e}_{v}+\omega_{n(c, v)}^{2} e_{v} \\
& =\left(\varphi-\widehat{\varphi}_{1}\right)+2 \xi_{c, v} \omega_{n(c, v)} \dot{\tilde{e}}_{v}-K_{h} \widehat{\sigma}_{1} .
\end{aligned}
$$

The closed loop tracking error dynamics (16) are excited by three terms, from which the terms $\left(\varphi-\widehat{\varphi}_{1}\right)$ and $\dot{\tilde{e}}_{v}$ correspond to estimation errors of the high gain GPI observer (12), but as shown before, by a proper selection of the GPI observer gains, these errors might be arbitrarily small around a vicinity of zero. The term $-K_{h} \widehat{\sigma}_{1}$, on the other hand, cannot be neglected, since it recreates the feedback reflection from the tire system to the wheel system. This feedback force reflection is recreated by inducing a tracking error between the driver commanded angular position and the wheel angular position settled by the wheel DC motor. This fact establishes a commitment between reflecting forces from the tire system and forcing the tracking error $e_{v}$ to zero. Asymptotic convergence of the tracking error $e_{v}$ to a small vicinity around zero can be obtained by a proper selection of the gains $\xi_{c, v}$ and $\omega_{n(c, v)}$.

The feedback reflection of the estimated disturbance term $\widehat{\sigma}_{1}$ from the steering rack subsystem to the steering wheel subsystem yields the haptic loop in the form of a feedback signal proportional to the lumped perturbation torques present at the rack system. This recreates, to the driver, the forces and dynamical effects of torques affecting the steering rack and tire mechanism. The gain $K_{h}$ weighs the amount of haptic feedback to the driver and can be related to driving sensation and feeling of the road conditions.

Remark 2. Note that in this proposed approach the driver "feels" proportional forces and disturbances present at the steering rack and tire, with proportional gain $K_{h}$, which is the simplest feedback situation. In works such as [12], active modification of the handling and steering system are proposed based on composed functions or adaptive gains. Such active feedback adjustment is common in vehicles where different stiffness is felt at low and high vehicle speed, as well as a modification of the steering angle ratio between steering wheel and tire is induced. Such modifications can be introduced through the proposed controller (15) by defining a feedback function of the estimated variable $\widehat{\sigma}_{1}$.

3.2. Steering Rack Controller and Observer. From Figures 2, 3,4 , and 5 , it is straightforward to find out the similarities between the steering wheel subsystem and the rack and tire subsystem. Due to the similarities between both subsystems, also a high gain observer and PD controller with disturbance rejection similar to (12) and (15) are proposed for the steering rack and tire subsystem. Thus, based on the dynamic model of the steering rack subsystem (5), the nonlinear terms and uncertain dynamics are substituted by a time-variant disturbance term $\sigma(t)$. Then, the state space dynamic model (5) is represented as in (17), with states $y_{1}=\theta_{r}$ and $y_{2}=\dot{\theta}_{r}$ :

$$
\begin{gathered}
\dot{y}_{1}=y_{2}, \\
\dot{y}_{2}=\frac{N_{r} k_{1}}{J_{r e} R_{r}} V_{r}(t)+\sigma(t)
\end{gathered}
$$

with

$$
\begin{aligned}
\sigma(t)=-\frac{1}{J_{r e}}\{ & \left(B_{T r}+B_{r}\right) \dot{\theta}_{r} \\
& \left.+\left(F_{T r}+C_{r}\right) \operatorname{sign}\left(\dot{\theta}_{r}\right)+\frac{N_{r}^{2} k_{1} k_{2}}{R_{r}} \dot{\theta}_{r}+\tau_{\mathrm{SAT}}\right\} .
\end{aligned}
$$

For the steering rack and tire subsystem, the equality established by (18) is enforced by the proposed model (5), if other dynamic effects or perturbations are to be considered, these phenomena would be included in the term $\sigma(t)$ as well, such that the approximated linear model (17) remains valid in local basis. The term $\sigma(t)$ would be estimated by a high gain GPI observer (19), its estimate is denoted by the variable $\widehat{\sigma}_{1}$ :

$$
\begin{aligned}
& \dot{\hat{y}}_{1}=\widehat{y}_{2}+\lambda_{7 r}\left(y_{1}-\widehat{y}_{1}\right) \\
& \dot{\hat{y}}_{2}=\frac{N_{r} k_{1}}{J_{r e} R_{r}} V_{r}(t)+\widehat{\sigma}_{1}+\lambda_{6 r}\left(y_{1}-\widehat{y}_{1}\right), \\
& \dot{\hat{\sigma}}_{1}=\widehat{\sigma}_{2}+\lambda_{5 r}\left(y_{1}-\widehat{y}_{1}\right) \\
& \dot{\hat{\sigma}}_{2}=\widehat{\sigma}_{3}+\lambda_{4 r}\left(y_{1}-\widehat{y}_{1}\right) \\
& \dot{\hat{\sigma}}_{3}=\widehat{\sigma}_{4}+\lambda_{3 r}\left(y_{1}-\widehat{y}_{1}\right) \\
& \dot{\hat{\sigma}}_{4}=\widehat{\sigma}_{5}+\lambda_{2 r}\left(y_{1}-\widehat{y}_{1}\right) \\
& \dot{\hat{\sigma}}_{5}=\widehat{\sigma}_{6}+\lambda_{1 r}\left(y_{1}-\widehat{y}_{1}\right) \\
& \dot{\hat{\sigma}}_{6}=\lambda_{0 r}\left(y_{1}-\widehat{y}_{1}\right) .
\end{aligned}
$$

The observer estimation error $\widetilde{e}_{r}=y_{1}-\widehat{y}_{1}$ satisfies the dynamics given by

$$
\tilde{e}_{r}^{(8)}+\lambda_{7 r} \widetilde{e}_{r}^{(7)}+\cdots+\lambda_{2 r} \ddot{\tilde{e}}_{r}+\lambda_{1 r} \dot{\tilde{e}}_{r}+\lambda_{0 r} \widetilde{e}_{r}=\sigma^{(6)} .
$$

The convergence properties of the estimation error at the steering rack $\widetilde{e}_{r}$ to a small vicinity of the origin can be established in a similar manner as for the steering wheel subsystem. This is assigning the associated characteristic polynomial to a suitable polynomial with known adequate roots, as in (14), ensuring faster observer dynamics than the controller dynamics.

Based on the estimated angular velocity of the steering rack subsystem $\widehat{y}_{2}$ along with the perturbation estimate $\widehat{\sigma}_{1}$, an active disturbance rejection control is synthesized in the form of a PD controller with a perturbation term rejection. This controller is proposed in (21). Notice that the reference 


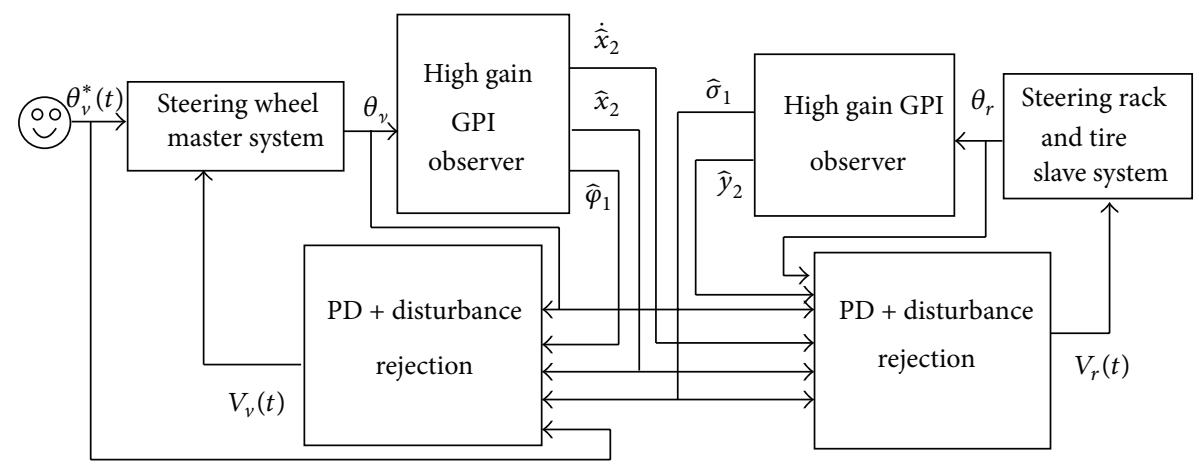

FIGURE 7: SBW schematic interconnections.

trajectory for the PD steering rack controller corresponds to the steering wheel angle $\theta_{v}(t)$ and the angular velocity and acceleration estimates $\widehat{x}_{2}$ and $\dot{\bar{x}}_{2}$, which are obtained by the high gain GPI observer (12). Therefore, the closed loop system can be seen as a master-slave system with a haptic loop and bilateral communication. The haptic loop is due to feedback reflecting the estimate of the steering rack perturbation term $\widehat{\sigma}_{1}$ to the steering wheel PD control, given by (15). Thus, through the steering wheel the human operator is able to feel the dynamic phenomena that affect the steering rack and tire:

$$
\begin{aligned}
V_{r}(t)=-\frac{J_{r e} R_{r}}{k_{1} N_{r}}[ & \widehat{\sigma}_{1}+\omega_{n(c, r)}^{2}\left(\theta_{r}-\theta_{v}(t)\right) \\
& \left.+2 \xi_{c, r} \omega_{n(c, r)}\left(\widehat{y}_{2}-\widehat{x}_{2}\right)-\dot{\bar{x}}_{2}\right] .
\end{aligned}
$$

By defining the tracking error at the steering rack and tire subsystem as $e_{r}=\theta_{r}-\theta_{v}$, then the closed loop dynamics formed by (17) with the controller (21) and the high gain observer (19) are given by the nonhomogeneous linear equation

$$
\begin{aligned}
\ddot{e}_{r} & +2 \xi_{c, r} \omega_{n(c, r)} \dot{e}_{r}+\omega_{n(c, r)}^{2} e_{r} \\
& =\left(\sigma-\widehat{\sigma}_{1}\right)+2 \xi_{c, r} \omega_{n(c, r)}\left(\dot{\tilde{e}}_{v}-\dot{\tilde{e}}_{r}\right)-\ddot{\tilde{e}}_{v} .
\end{aligned}
$$

The closed loop tracking error dynamics (22) are excited by estimation errors of the high gain GPI observers (12) and (19); nevertheless, by a proper selection of the GPI observer gains, these errors might be arbitrarily small around a vicinity of zero. Thus asymptotic convergence of the tracking error $e_{r}$ to a small vicinity around zero can be obtained by a proper selection of the gains $\xi_{c, r}$ and $\omega_{n(c, r)}$.

Notice that the full control system given by the controllers (15) and (21) together with the high gain GPI observers (12) and (19) only require measurement of the steering wheel angle $\theta_{v}$ and the tire orientation angle $\theta_{r}$. It is also important to point out that the proposed approach is based on the input gain of the systems; thus minimum knowledge of the dynamic models is required. Figure 7 shows a schematic representation of the proposed SBW system and the interconnections between the steering wheel and steering rack and tire subsystem.

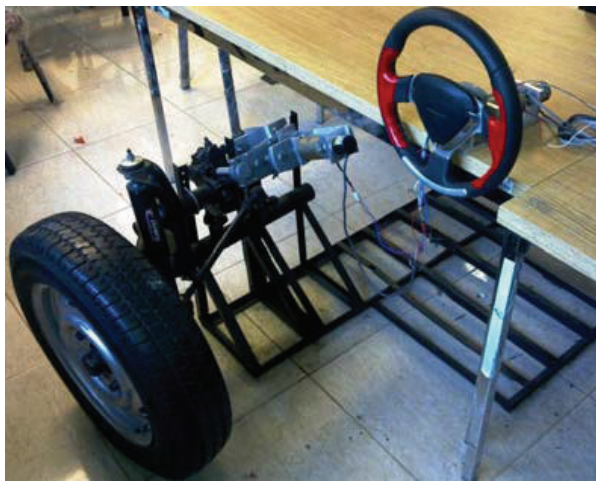

FIGURE 8: SBW experimental platform.

\section{Experimental Results}

For experimental purposes, a low cost platform has been built (see Figure 8). The platform is provided with a steering wheel, and half of the steering rack of a beetle VW vehicle, including suspension system and tire. This type of steering system is still available in economic commercial cars. The steering column was modified as shown in Figures 2 and 4, including 2 DC motors and encoders. The communication and control are programmed in a PC with Windows as operative system.

The whole control approach of the SBW haptic system, PD, and high gain GPI observer (12) and (15) for the steering wheel and (19) and (21) for the steering rack are programmed using general blocks of Simulink available in MATLAB. The electronics and mechanical components of the experimental platform are listed as follows. The angular positions on the steering wheel and on the rack are measured by incremental encoders OMRON, model E6B2-CWZ1X, 2000PPR, 0.5 M. The PC is equipped with a data acquisition card Sensoray model 626, which is programmed to work with a sampling period of 0.0005 seconds. Two operational amplifiers, model STK4050 II, are used to condition the control voltages $V_{v}(t)$ and $V_{r}(t)$ that are sent to the DC motors Nisca Motor model NC5475B. Each motor is connected to a gearbox, such that for the steering wheel a reduction ratio of $N_{v}=16$ is obtained, and for the steering rack the reduction ratio is $N_{r}=48$. Figure 9 shows a schematic connection of the SBW haptic system. 


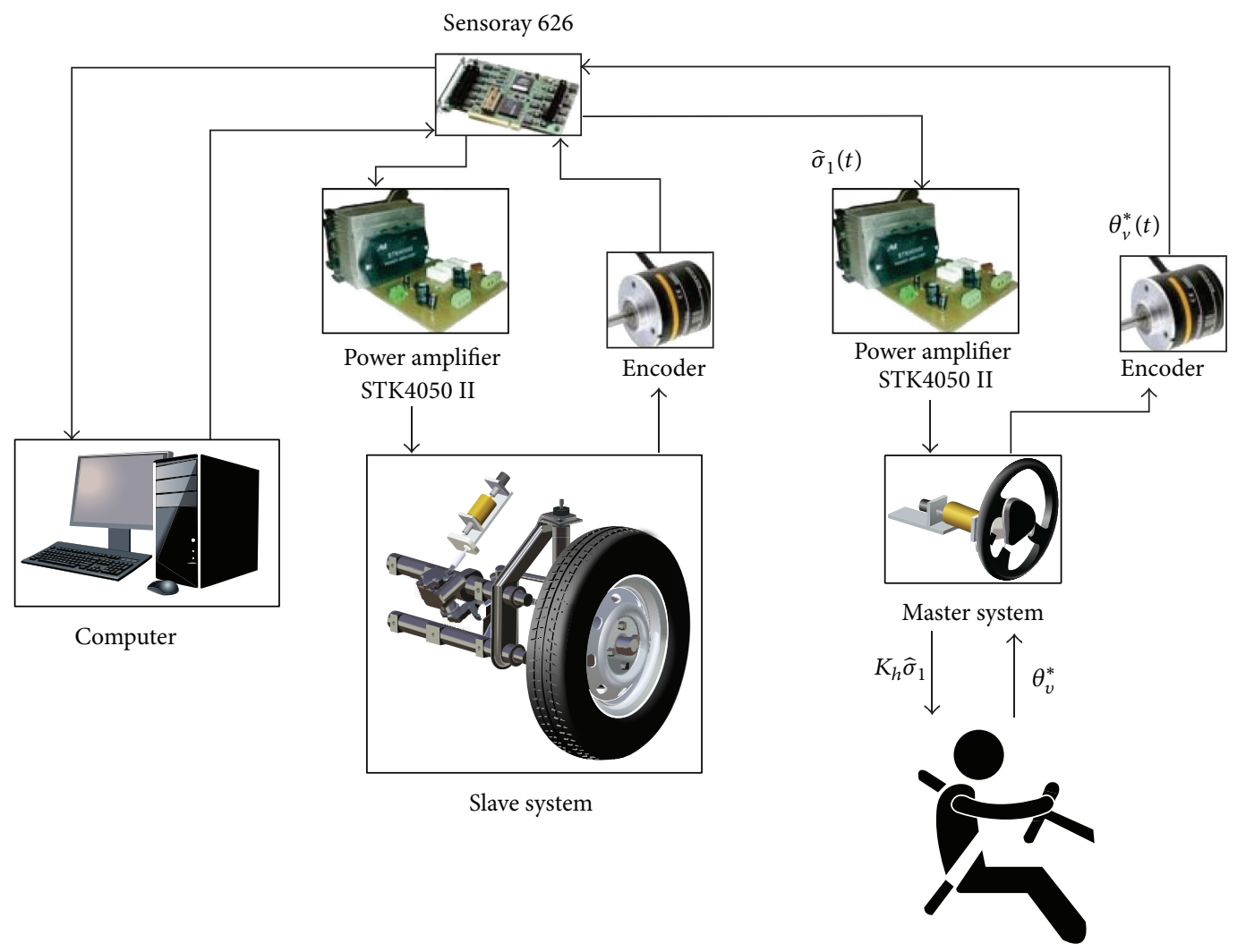

FIGURE 9: Schematic diagram of the experimental SBW.

Following the results from $[18,19]$, selection of the control and observer gains should imply that the observer error dynamics must be faster than the controller dynamics. All gains were tuned by using (14), by considering pure real roots and taking into account the performance of the tracking errors $e_{v}$ and $e_{r}$. For the master subsystem the control gains involved in (15) are $\xi_{c, v}=1.01, \omega_{n(c, v)}=5.92$, and for its GPI observer (12) $\xi_{\mathrm{obs}, v}=2.2, \omega_{n(\mathrm{obs}, v)}=15.2$ meanwhile, for the steering rack (slave subsystem) the control gains, see (21), they are $\xi_{c, r}=2.53, \omega_{n(c, r)}=5.92$, and for the GPI observer (19) the gains are $\xi_{\mathrm{obs}, r}=10, \omega_{n(\mathrm{obs}, r)}=25$.

Two cases are compared: first the SBW is tested without traction, and second tire traction at a speed of $9.4 \mathrm{~m} / \mathrm{s}$ $(33.84 \mathrm{~km} / \mathrm{hr})$ is supplied. This comparison is to look after how tire velocity affects the aligning forces and thus the reaction forces presented at tire and steering rack subsystem. The same platform as sketched in Figure 9 is used for both cases, with and without tire traction; only the motor in charge of traction is turned on or off; this emulates steering the vehicle when being parked and in motion. It is also important to emphasize that the controller and observers are the same in both cases; that is, nothing is changed in their structure. This fact implies a realistic and robust application of the proposed approach in a vehicle, such that the same controller and observers work for all possible vehicle scenarios. The control gains that are used in both cases are those described at previous paragraph, which were tuned for the case without tire traction. For the sake of comparison first the results without traction are presented; then the results with traction are shown.

4.1. SBW System without Tire Traction. This case emulates steering the vehicle when being parked, such that greater effort is required to steer the tire than when being in motion. In some commercial vehicles, a larger steering ratio, between steering wheel and tire, is set when the vehicle moves at low speed or is standstill. This produces a less stiff or softer steering wheel sensation. In this experiment and for comparison purposes the same steering ratio and feedback gain for the haptic loop $\left(K_{h}\right)$ are kept for traction and without traction cases.

The convergence between the steering rack angle (tire angle orientation) $\theta_{r}$ and the human desired angular position given by the desired steering wheel angle $\theta_{v}^{*}$ allows concluding good tracking and performance of the SBW system; see Figure 10. This is further supported by the tracking error $e=$ $\theta_{r}-\theta_{v}^{*}$ that converges to a small vicinity around zero; see Figure 11. Thus, it can be concluded that the high gain GPI observer (19) properly estimates the disturbance signal at the steering rack and tire $\widehat{\sigma}_{1}(t)$; see Figure 12 . Therefore the PD plus disturbance compensation (21) is able to reject the perturbation signal effects. On the other hand, feedback of the dynamic perturbation phenomena $\widehat{\sigma}_{1}(t)$ from the steering rack to the steering wheel generates a haptic loop such that the operator "feels" the dynamic perturbations that affect the 


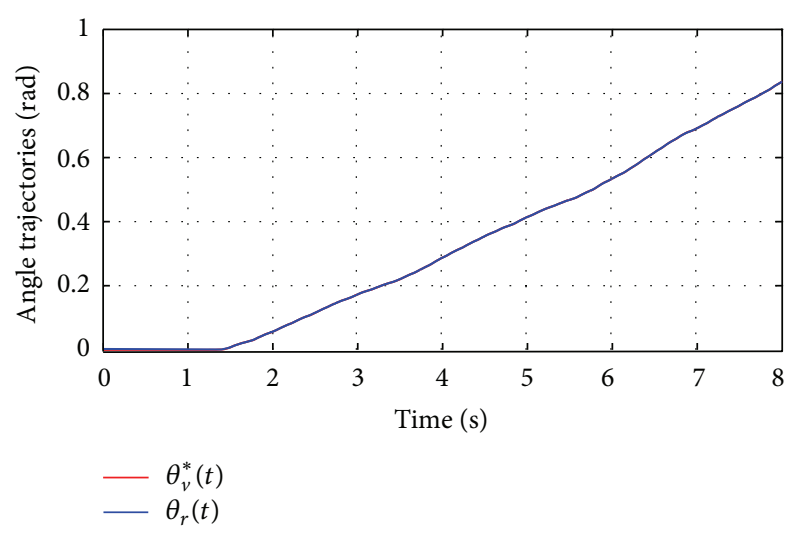

FIGURE 10: Rack angular position and its desired human reference $\theta_{v}(t)^{*}$ without tire traction.

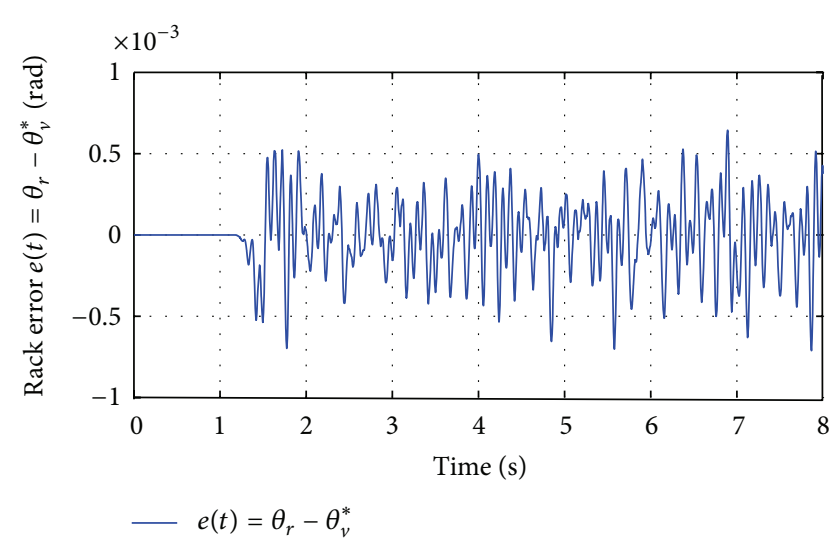

FIGURE 11: Rack tracking error $e_{r}(t)$ without tire traction.

steering rack and tire. The DC motor voltage on the steering rack subsystem $V_{r}(t)$ required to properly track the rack angular reference is shown in Figure 13.

4.2. SBW System with Tire Traction. This case emulates steering the vehicle when being in motion, such that less effort is required to steer the tire than when being parked. In some commercial vehicles a smaller steering ratio, between steering wheel and tire, is set as higher the vehicle speed, as well as a stiffer steering wheel feeling, this is the so called "sport" mode. Although such adaptive response of the SBW can be considered in the proposed controller (15), in this experiment and for comparison purposes the same steering ratio and feedback gain for the haptic loop $\left(K_{h}\right)$ are kept for traction and without traction cases.

When tire traction is considered $(9.4 \mathrm{~m} / \mathrm{s})$, convergence between the steering rack angle $\theta_{r}$ and the human desired angular position $\theta_{v}^{*}$ allows concluding good tracking and performance of the SBW system; see Figure 14. This is further supported by the tracking error $e_{r}=\theta_{r}-\theta_{v}^{*}$ that converges to a small vicinity around zero; see Figure 15 . Thus, the high gain GPI observer (19) properly estimates the perturbation signal

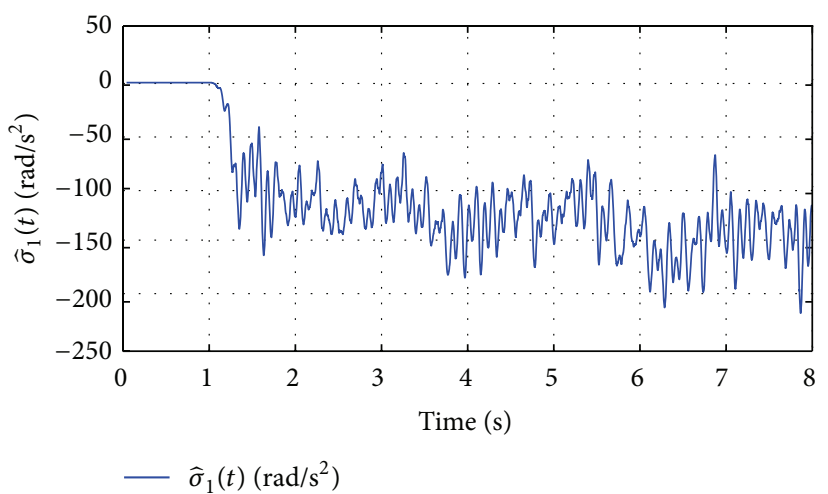

FIGURE 12: Disturbance steering rack signal $\widehat{\sigma}_{1}(t)$ without tire traction.

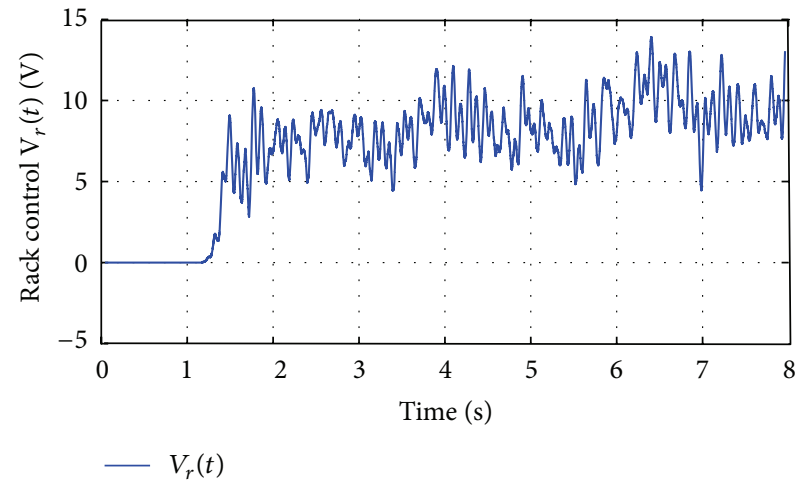

FIGURE 13: Voltage at the steering rack DC motor $V_{r}(t)$ without tire traction.

at the steering rack and tire $\widehat{\sigma}_{1}(t)$; see Figure 16 . Thus, the PD plus disturbance compensation (21) is able to reject the perturbation signal effects. On the other hand, feedback of the disturbance phenomena $\widehat{\sigma}_{1}(t)$ from the steering rack to the steering wheel generates a haptic loop such that the operator "feels" the dynamic perturbation that affect the steering rack and tire. The DC motor voltage on the steering rack subsystem $V_{r}(t)$ required to properly track the rack angular reference is shown in Figure 17.

4.3. Comparison Results and Discussion. It is important to point out that the commanded steering wheel reference was provided by a human operator steering the wheel. Therefore, although the same desired trajectory was intended, there are differences on such reference; see Figures 10 and 14. Nevertheless, some conclusions can be drawn from the comparison study.

In both cases, with and without tire traction, the performance of the SBW is rather acceptable; that is, the tracking error $e=\theta_{r}-\theta_{v}^{*}$ converges to a small vicinity around zero, as predicted by the closed loop error dynamics (22); that is, $\theta_{r} \rightarrow \theta_{v} \rightarrow \theta_{v}^{*}$; see Figures 11 and 15. It is important to note that the control and observer gains were tuned for the without tire traction case; nevertheless, the magnitude and performance of the tracking error $e=\theta_{r}-\theta_{v}^{*}$ is very similar 


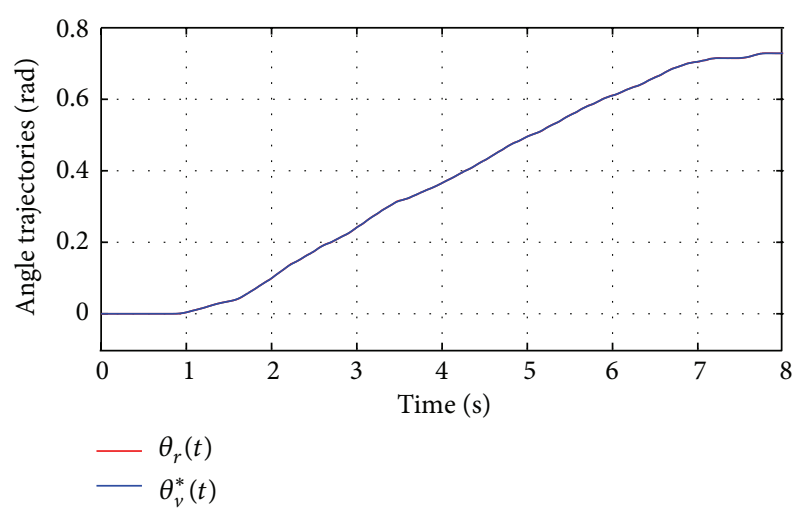

FIGURE 14: Rack angular position and its desired human reference $\theta_{v}(t)^{*}$ with tire traction.

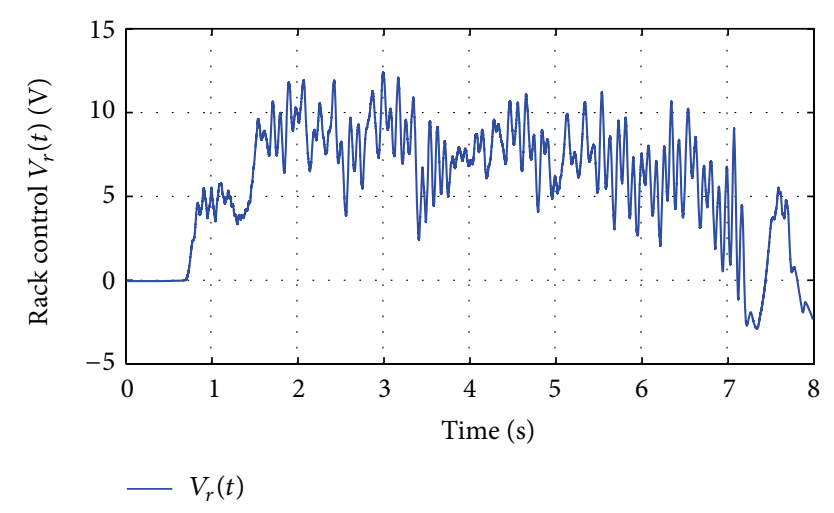

FIGURE 15: Rack tracking error $e_{r}(t)$ with tire traction.

for both cases. Therefore, it can be concluded that uncertainty and dynamic perturbations are properly estimated and compensated by the high gain GPI observers in both cases, with and without traction.

Even though similar tracking error convergence $e=\theta_{r}-$ $\theta_{v}^{*}$ is obtained for both cases, there are relevant differences on magnitude and shape of the estimated perturbation term $\widehat{\sigma}_{1}(t)$ (Figures 12 and 16), as well as for the control action $V_{r}(t)$ (Figures 13 and 17). These differences are due to the effects of tire traction, particularly, aligning torques and road-tire friction. The estimated perturbation signals $\widehat{\sigma}_{1}(t)$ show that for the case with traction, the perturbation torque is smaller in magnitude that for the without tire traction. This fact translates in the driver feeling less effort to steer the wheel when there is traction, than without traction. This "feeling" is common when driving a vehicle at high speed, when less effort is required to steer, compared to the steering effort when the vehicle speed is very low or even being parked.

Notice that some commercial vehicles actively adapt the steering ratio, between steering wheel and tire, and stiffness steering wheel feeling, as function of the vehicle speed, for example, the parking and sport mode. Although such adaptive response of the SBW can be considered in the proposed controller (15), in the comparison study here carried out the same steering ratio and feedback gain for the haptic loop $\left(K_{h}\right)$

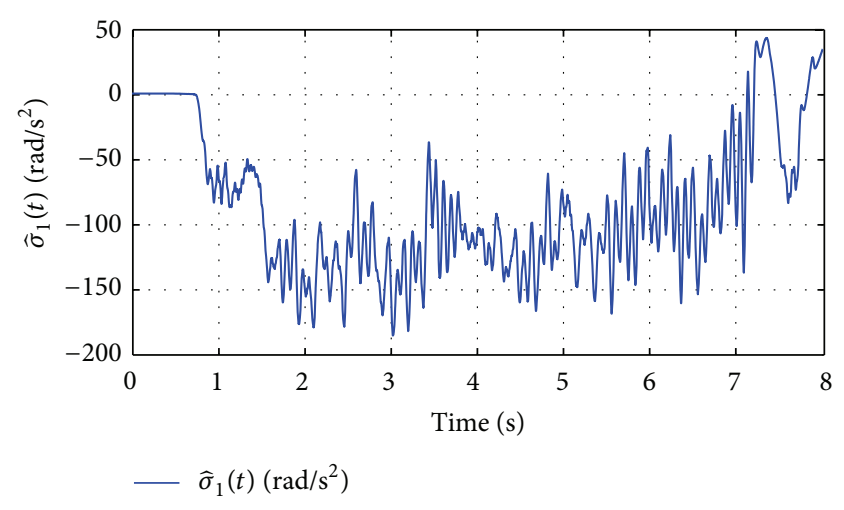

FIGURE 16: Disturbance steering rack signal $\widehat{\sigma}_{1}(t)$ with tire traction.

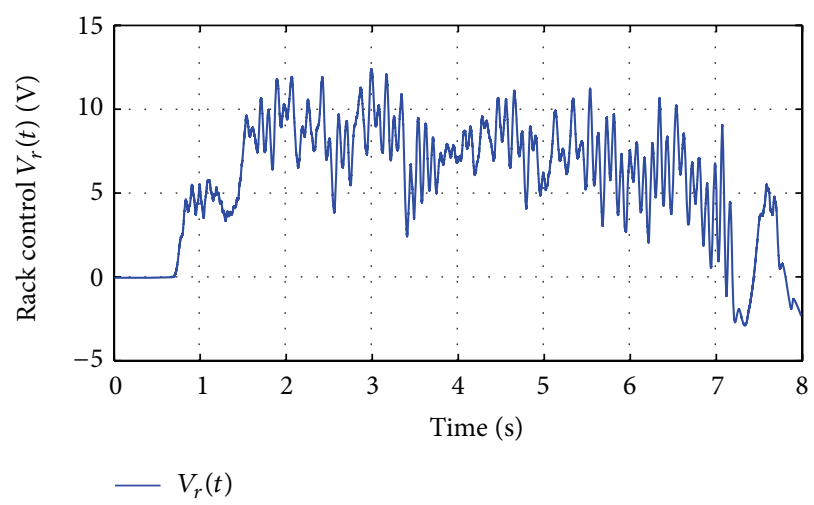

FIGURE 17: Voltage at the steering rack DC motor $V_{r}(t)$ with tire traction.

are kept for traction and without traction cases. Adaptive behavior of the proposed SBW haptic system can be considered by modifying the feedback of the disturbance and dynamic effects of the steering rack and tire.

\section{Conclusions and Perspectives}

This paper has presented a haptic steering by wire system based on high gain GPI observers. The high gain GPI technique requires a minimum amount of information from the dynamic model; only the nonlinear system input gain is required; as for sensors only encoders are employed, which is an advantage compared to other techniques of SBW systems that required specialized sensors. The tracking error between the desired angle settled by the human operator, the steering wheel angle, and the tire orientation angle can be made arbitrarily small by a proper gain selection.

The technique is capable of estimating the perturbation inputs and the uncertain terms that affect the SBW dynamics. In particular, the SBW system represents a twofold application of the GPI observer: on the one hand it helps in rejection or compensating the perturbation in each subsystem; on the other hand the estimated perturbations on the steering rack are fed back to the steering wheel to yield a haptic system, including tire self-aligning torque. 
It is worth to notice that the high gain GPI observer together with the PD control relies only on measurements of an encoder, thus highly diminishing the use of sensors. Then the proposed SBW system behaves as a self-contained sensor for steering rack and tire forces. To validate this last conclusion, as future work, the installation of force, acce leration, and other sensors are being considered. This will allow establishing clear comparisons between the estimated perturbation and measurements on the experimental platform.

\section{Appendix}

\section{Ultra Models}

Considering the single-input single-output, $n$-dimensional perturbed nonlinear system is given by (A.1). The initial conditions at $t_{0}$ are given by the set $\mathscr{Y}_{0}=\left\{y_{0}, \dot{y}_{0}, \ldots, y_{0}^{(n-1)}\right\}$. Assume that the input $u=u(t)$ and the gain input function $\phi(t, y)$ are perfectly known. The nonlinear input gain function $\phi(t, y(t))$ is assumed to be bounded and uniformly bounded away from zero:

$$
y^{(n)}=\psi\left(t, y, \dot{y}, \ldots, y^{(n-1)}\right)+\phi(t, y) u .
$$

Definition A.1. Let $\xi(t)$ be a time function, which represents the additive nonlinear term $\psi\left(t, y, \dot{y}, \ldots y^{(n-1)}\right)$ in (A.1), particularized for $y=y(t)$, where the function $y(t)$ is the solution of (A.1) for a given input function $u(t)$ and for a set of initial conditions $\mathscr{Y}_{0}$. That is,

$$
\xi(t)=\psi\left(t, y(t), \dot{y}(t), \ldots, y^{(n-1)}(t)\right) .
$$

Defining a global ultra model of the system (A.1), the system represented by (A.3), subject to the corresponding set of initial conditions $\mathscr{Z}_{0}$ satisfies $\mathscr{Z}_{0}=\mathscr{Y}_{0}$ :

$$
z^{(n)}=\xi(t)+\phi(t, z(t)) u(t) .
$$

The term $\xi(t)$ in (A.3) is obviously a priori unknown; nevertheless, it is algebraically observable in the sense of Diop and Fliess [26]. The uncertainty in $\xi(t)$ is basically due to parameters and unknown nonlinearities in the term $\psi(\cdot)$ in (A.1). It may also represent exogenous perturbations which are unmodeled. As a time function, $\xi(t)$, and a finite number of its time derivatives (e.g., $p$ derivatives) are assumed to be uniformly absolutely bounded. According to Gliklikh [27], without this hypothesis, there is not any solutions to the original system. In open loop, the nominal control input $u^{*}(t)$, corresponds, due to differential flatness of the system [28], to an smooth output reference trajectory $y^{*}(t)$.

Notice that for any bounded input function $u(t)$, and a given initial condition set $\mathscr{Y}_{0}$, the solution of system (A.1) $y(t)$ satisfies, as a time function, the identity (A.4) [29]:

$$
y^{(n)}=\psi(t, y, \dot{y}), \ldots, y^{(n-1)}+\phi(t, y) u(t) .
$$

Theorem A.2. The system (A.3) is trajectory equivalent to the nonlinear system (A.1). That is, both systems present exactly the same solution trajectories $y(t)=z(t)$ for all $t$, for a particular common initial condition set $\mathscr{Y}_{0}$.
Proof. Define $\varepsilon=y(t)-z(t)$; then (A.3) and (A.1) imply that $\varepsilon$ satisfies $\varepsilon^{(n)}(t)=[\phi(t, y(t))-\phi(t, z(t))] u(t)$, with zero initial conditions, $\varepsilon^{(k)}\left(t_{0}\right)=0, k=0,1, \ldots, n-1$. Meanwhile, $\varepsilon^{(n)}\left(t_{0}\right)=0$ and $\varepsilon^{(n+j)}\left(t_{0}\right)=0$, for all $j$. Therefore, it follows that $\varepsilon(t)=0$ for all $t$; thus systems (A.3) and (A.1) are trajectory equivalent.

The consequence of the previously stated fact is that the nonlinear systems (A.1) can be seen as the linear perturbed system (A.3), including the exogenous presence of timevariant term: $\xi(t)=\psi\left(t, y(t), \ldots, y^{(n-1)}(t)\right)$. These two systems (A.1) and (A.3) are identical on the precise sense that their trajectories are the same for any time interval. Thus, distinction between $z(t)$ and $y(t)$ becomes irrelevant. Any system of the form (A.1) can be examined through the equivalent trajectory of system (A.3), which can be seen, without any ambiguity, as in

$$
y^{(n)}=\xi(t)+\phi(t, y) u
$$

The system (A.5) which is trajectory equivalent to the original nonlinear system (A.1) lacks the state timedependent additive nonlinear term. In practice, the value of the time-dependent nonlinearities, lumped in the term $\xi(t)$, might be unknown and thus it can be seen as a perturbation to the system. The use of (A.5) greatly simplifies the simultaneous observation problem of the nonlinear perturbation, which is state dependent, and of the state of the system (A.1) itself, based on the input $u$ and output $y$ signals.

\section{Conflict of Interests}

The authors declare that there is no conflict of interests regarding the publication of this paper.

\section{Acknowledgment}

The authors acknowledge support from CONACyT, Mexico, through Project 133527.

\section{References}

[1] R. Bosch, Bosch Automotive Handbook, John Wiley \& Sons, Chicester, UK, 7th edition, 2008.

[2] J. Reimpell, H. Stoll, and J. Betzler, The Automotive Chassis: Engineering Principles, Butterworth-Heinemann, London, UK, 2001.

[3] J. Y. Wong, Theory of Ground Vehicles, John Wiley \& Sons, New York, NY, USA, 1978.

[4] T. K. Garrett, K. Newton, and W. Steeds, The Motor Vehicle, Butterworth-Heinemann, London, UK, 2001.

[5] S. Amberkar, M. Kushion, K. Eschtruth, and F. Bolourchi, "Diagnostic development for an electric power steering system," SAE Technical Paper 2000-01-0819, 2000.

[6] D. Peter and R. Gerhard, "Electric power steering - the first step on the way to steer by wire," SAE Technical Paper 1999-01-0401, 1999.

[7] J. W. Post, Modeling, simulation and testing of automobile power steering system for the evaluation of on-center handling [Ph.D. 
thesis], Graduate School Clemson University, Clemson, SC, USA, 1995.

[8] J. Kim and J. Song, "Control logic for an electric power steering system using assist motor," Mechatronics, vol. 12, no. 3, pp. 447459, 2002.

[9] A. Zaremba and R. I. Davis, "Dynamic analysis and stability of a power assist steering system," in Proceedings of the American Control Conference, pp. 4253-4257, June 1995.

[10] A. T. Zaremba, M. K. Liubakka, and R. M. Stuntz, "Control and steering feel issues in the design of an electric power steering system," in Proceedings of the American Control Conference (ACC '98), pp. 36-40, June 1998.

[11] A. E. Cetin, M. A. Adli, D. E. Barkana, and H. Kucuk, "Implementation and development of an adaptive steering-control system," IEEE Transactions on Vehicular Technology, vol. 59, no. 1, pp. 75-83, 2010.

[12] P. Yih and J. C. Gerdes, "Modification of vehicle handling characteristics via steer-by-wire," IEEE Transactions on Control Systems Technology, vol. 13, no. 6, pp. 965-976, 2005.

[13] A. Baviskar, J. R. Wagner, D. M. Dawson, D. Braganza, and P. Setlur, "An adjustable steer-by-wire haptic-interface tracking controller for ground vehicles," IEEE Transactions on Vehicular Technology, vol. 58, no. 2, pp. 546-554, 2009.

[14] A. Bertacchini, L. Tamagnini, and P. Pavan, "Force feedback in steer-by-wire systems: architecture and experimental results," in Proceedings of the International Symposium on Industrial Electronics 2006 (ISIE '06), pp. 3050-3055, IEEE, Montreal, Canada, July 2006.

[15] B.-H. Nguyen and J.-H. Ryu, "Direct current measurement based steer-by-wire systems for realistic driving feeling," in Proceedings of the International Symposium on Industrial Electronics (ISIE '09), pp. 1023-1028, Seoul, Republic of Korea, July 2009.

[16] L. Li, F. Wang, and Q. Zhou, "Integrated longitudinal and lateral tire/road friction modeling and monitoring for vehicle motion control," IEEE Transactions on Intelligent Transportation Systems, vol. 7, no. 1, pp. 1-19, 2006.

[17] C. Kim, K. Hong, W. Yoo, and Y. Park, "Tire-road friction estimation for a wheel-driven field robot," in Proceedings of the Society of Instrument and Control Engineers (SICE '08), pp. 782787, Tokyo, Japan, August 2008.

[18] B. Guo and Z. Zhao, "On convergence of the nonlinear active disturbance rejection control for MIMO systems," SIAM Journal on Control and Optimization, vol. 51, no. 2, pp. 1727-1757, 2013.

[19] W. Zhou, S. Shao, and Z. Gao, "A stability study of the active disturbance rejection control problem by a singular perturbation approach," Applied Mathematical Sciences, vol. 3, no. 10, pp. 491-508, 2009.

[20] H. Sira-Ramírez, "On the generalized PI sliding mode control of DC-to-DC power converters: a tutorial," International Journal of Control, vol. 76, no. 9-10, pp. 1018-1033, 2003.

[21] H. Sira-Ramirez, F. Gonzalez-Montanez, J. A. Cortes-Romero, and A. Luviano-Juarez, "A robust linear field-oriented voltage control for the induction motor: experimental results," IEEE Transactions on Industrial Electronics, vol. 60, no. 8, pp. 30253033, 2013.

[22] R. E. Bou Serhal and H. K. Khalil, "Application of the extended high gain observer to underactuated mechanical systems," in Proceedings of the American Control Conference (ACC '12), pp. 4727-4732, June 2012.

[23] Y. Yamaguchi and T. Murakami, "Adaptive control for virtual steering characteristics on electric vehicle using steer-by-wire system," IEEE Transactions on Industrial Electronics, vol. 56, no. 5, pp. 1585-1594, 2009.

[24] J. Im, F. Ozaki, N. Matsunaga, and S. Kawaji, "Control of steering-by-wire system using bilateral control scheme with passivity approach," in Proceedings of the International Conference on Control, Automation and Systems (ICCAS '07), pp. 1488-1493, October 2007.

[25] H. Khalil and L. Praly, "High-gain observers in nonlinear feedback control," International Journal of Robust and Nonlinear Control, vol. 24, no. 6, pp. 993-1015, 2014.

[26] S. Diop and M. Fliess, "Nonlinear observability, identifiability, and persistent trajectories," in Proceedings of the 30th IEEE Conference on Decision and Control, pp. 714-719, Brighton, UK, December 1991.

[27] Y. E. Gliklikh, "Necessary and sufficient conditions for globalin-time existence of solutions of ordinary, stochastic, and parabolic differential equations," Abstract and Applied Analysis, vol. 2006, Article ID 39786, 17 pages, 2006.

[28] H. Sira-Ramirez and S. Agrawal, Differentially Flat Systems, Marcel Dekker, New York, NY, USA, 2004.

[29] L. S. Pontryagin, Differential Equations, Addison-Wesley, Reading, Mass, USA, 1962. 


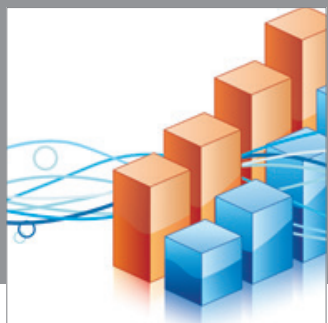

Advances in

Operations Research

mansans

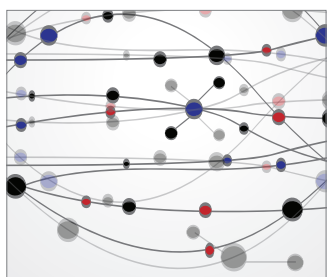

The Scientific World Journal
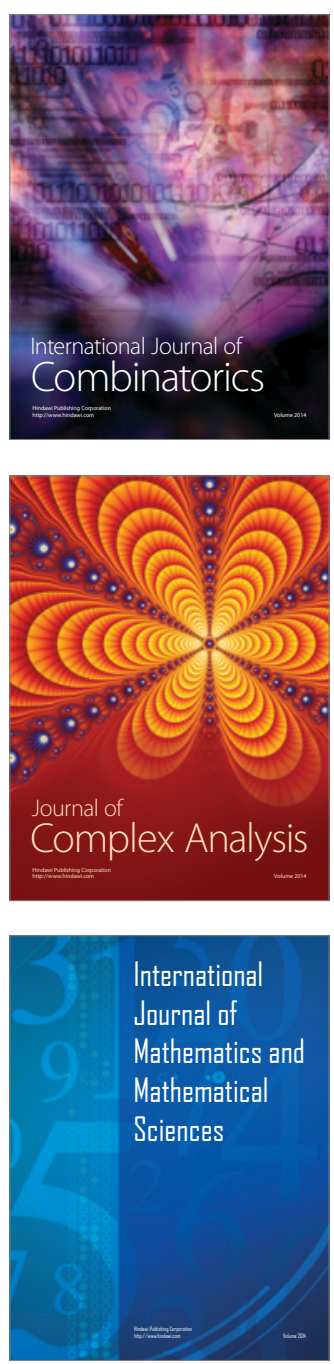
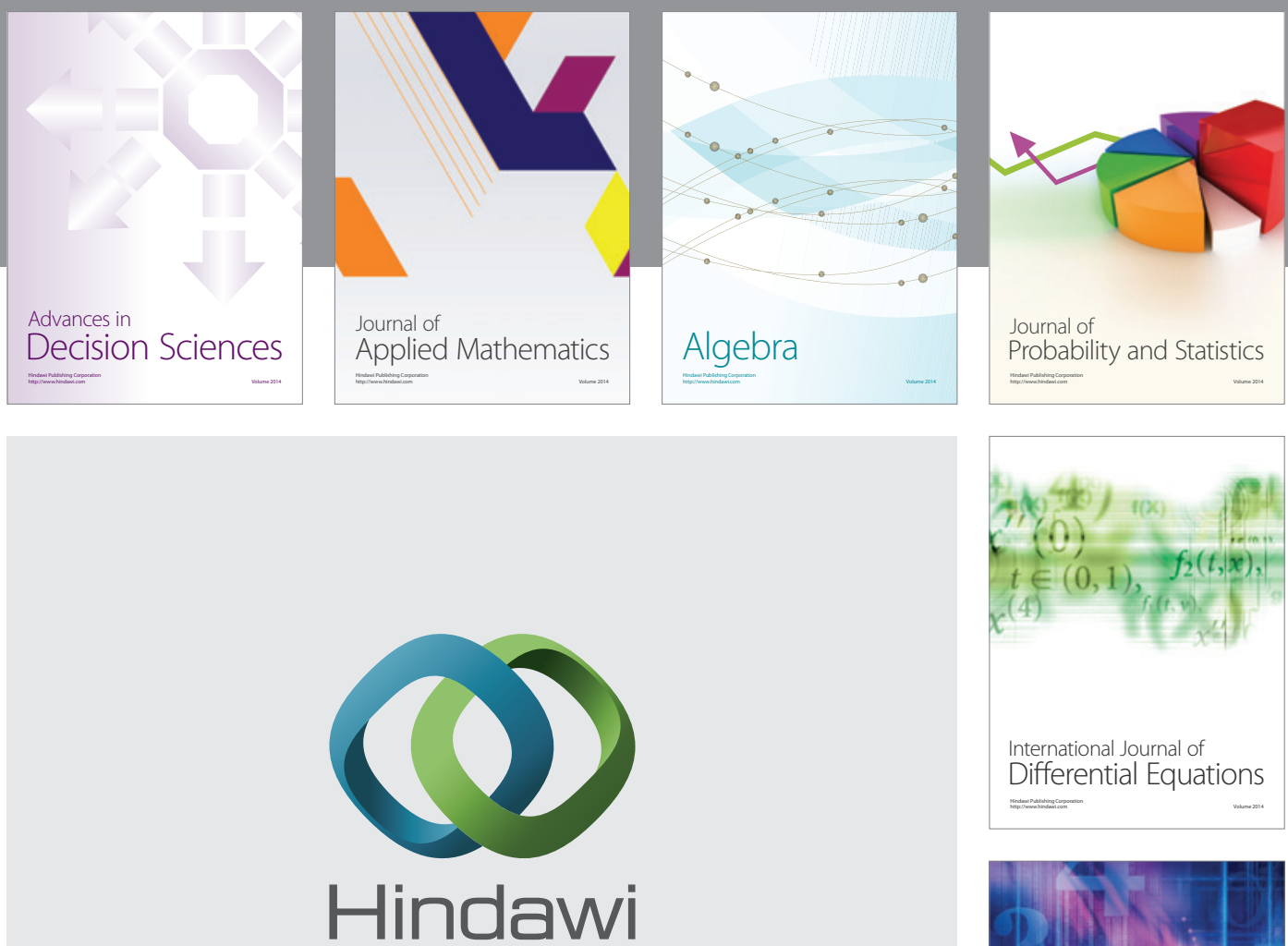

Submit your manuscripts at http://www.hindawi.com
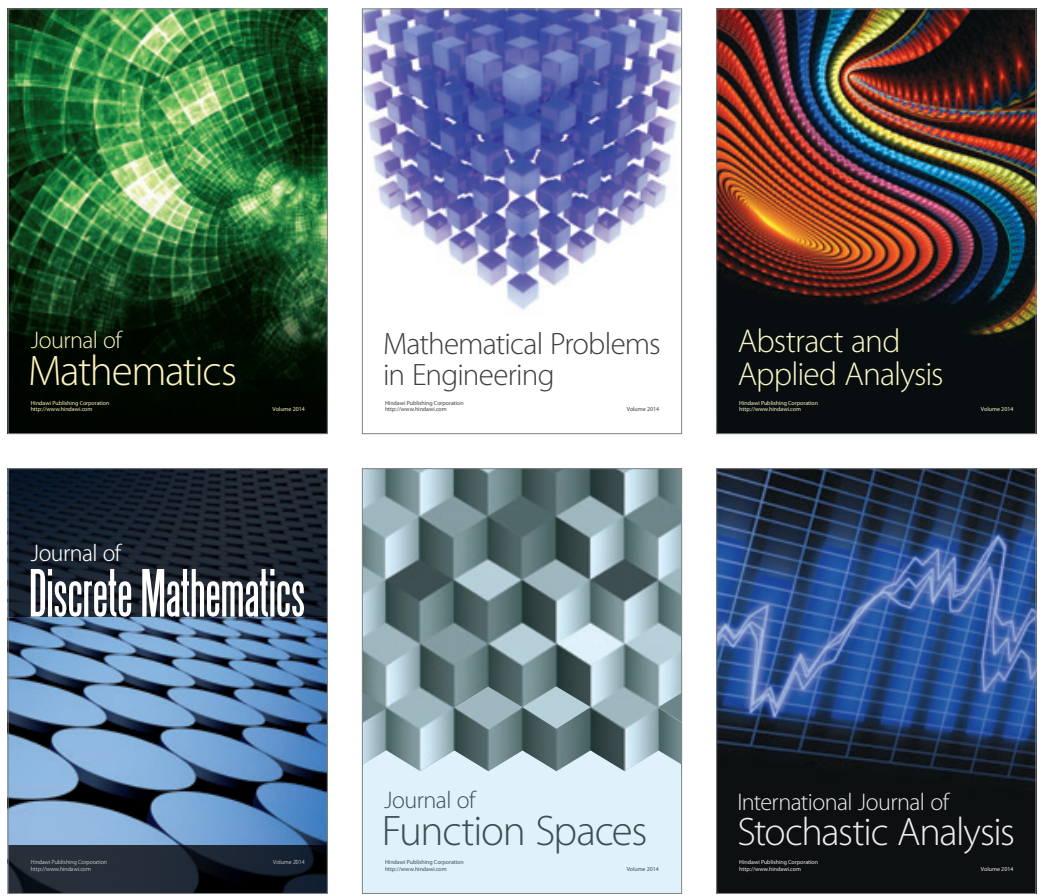

Journal of

Function Spaces

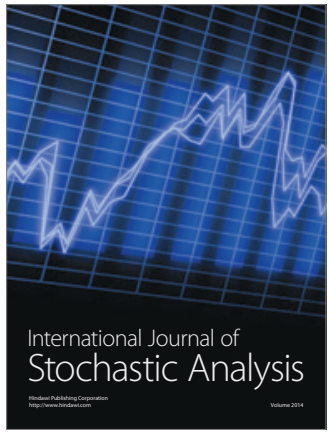

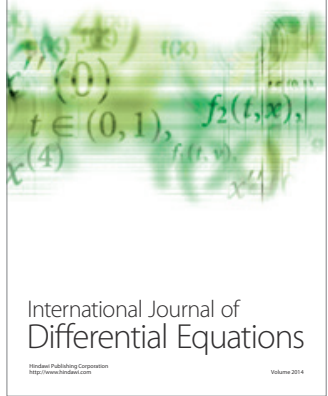
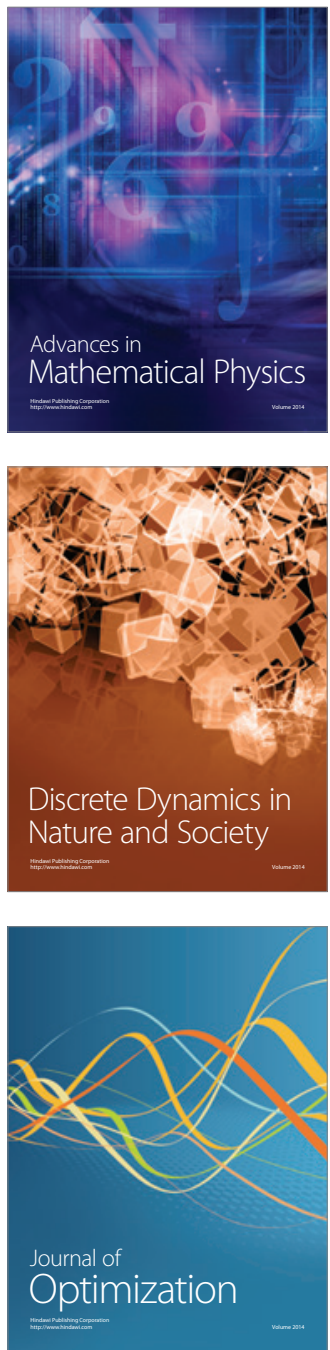